\title{
Impact of new manufacturing technologies on the petrochemical industry in the United States: a methane-to-aromatics case study
}

\author{
Supporting Information
}

Sean E. DeRosa* and David T. Allen

Center for Energy and Environmental Resources, University of Texas at Austin, 10100 Burnet Road, Bldg. 133, R7100, Austin, TX 78758, United States

*Author to whom correspondence should be addressed (E-mail: sean.derosa@utexas.edu)

\section{Ancillary Supply Chain Effects}

Besides the technology shifts caused by the new process, a change in benzene production cost introduces cost effects on other chemicals, even if their production technologies did not change. When the process is first introduced at Point B, coke, naphthalene, anthraquinone, and syndiotactic polystyrene show a decrease in shadow price. By Point D, when $97.8 \%$ of benzene is produced by the new process, 83 chemicals show a change in shadow price. The list of shadow price changes from Point $\mathrm{C}$ to Point $\mathrm{D}$ is shown in Table $\mathrm{S} 1$.

Table S1. Change in chemical shadow prices as utilization of the methane-to-aromatics process increases from $0.67 \%$ to $97.8 \%$ of the benzene market (Point $\mathrm{C}$ to Point D).

\begin{tabular}{ll}
\hline Chemical & Change in Shadow Price (\%) \\
\hline naphthalene & $-1.0 \mathrm{E}+02$ \\
\hline perchloroethylene & $-1.0 \mathrm{E}+02$ \\
\hline toluene & $-1.0 \mathrm{E}+02$ \\
\hline$n$-butyl acetate & $-1.0 \mathrm{E}+02$ \\
\hline reformate, heart cut & -88 \\
\hline anthraquinone & -86 \\
\hline cyclohexane & -74 \\
\hline benzene & -71 \\
\hline$n$-heptane & -64 \\
\hline chlorobenzene & -58 \\
\hline cumene & -52 \\
\hline$o$-xylene & -52 \\
\hline ethylbenzene & -28 \\
\hline phthalic anhydride & -25 \\
\hline phenol & -23 \\
\hline maleic anhydride & -22
\end{tabular}




\begin{tabular}{ll} 
styrene & -22 \\
\hline cyclohexanone & -21 \\
\hline nitrobenzene & -21 \\
\hline nylon salt, 63\% solution & -21 \\
\hline cyclohexanol & -19 \\
\hline polystyrene, general purpose & -19 \\
\hline bisphenol A & -19 \\
\hline$o$-dichlorobenzene & -18 \\
\hline polystyrene, high impact & -15 \\
\hline adipic acid & -14 \\
\hline polystyrene, anionic & -13 \\
\hline aniline & -12 \\
\hline diphenyl carbonate & -12 \\
\hline polystyrene, expandable & -11 \\
\hline polyester, unsaturated & -11 \\
\hline catechol & -9.2 \\
\hline benzoic acid & -8.7 \\
\hline SAN resin & -8.5 \\
\hline methylene diphenylene isocyanate & -8.4 \\
\hline polycarbonate & -8.4 \\
\hline ABS resin & -7.6 \\
\hline epoxy, solid DGEBPA and BPA & -7.0 \\
\hline ammonium sulfate & -6.9 \\
\hline hydroquinone & -6.7 \\
\hline styrene-butadiene block copolymer & -6.5 \\
\hline acetone & -6.2 \\
\hline petroleum resin, C5 aliphatic & -5.3 \\
\hline diphenyl isophthalate & -5.0 \\
\hline propylene oxide & -4.9 \\
\hline nylon-6,6 chips & -3.5 \\
\hline elastomer, polyurethane & -2.5 \\
\hline acetic anhydride & -2.3 \\
\hline methyl isobutyl ketone & -1.9 \\
\hline styrene-butadiene rubber & -1.7 \\
\hline propylene glycol ethers & -1.1 \\
\hline polyacrylate pellets & -1.0 \\
\hline nitric acid, dilute & -0.27 \\
\hline peracetic acid & -0.25 \\
\hline polybutadiene & -0.12 \\
\hline polybutene-1 & $-9.3 \mathrm{E}-2$ \\
\hline acrylic acid, ester grade & $-7.0 \mathrm{E}-2$ \\
\hline isobutylene, high purity & $-6.7 \mathrm{E}-2$ \\
\hline
\end{tabular}




\begin{tabular}{ll} 
epoxy, liquid DGEBPA & $-4.1 \mathrm{E}-2$ \\
\hline methyl acrylate & $-3.8 \mathrm{E}-2$ \\
\hline ethyl acrylate & $-3.4 \mathrm{E}-2$ \\
\hline carbofuran & $-2.9 \mathrm{E}-2$ \\
\hline$t$-butanol, gasoline grade & $-2.6 \mathrm{E}-2$ \\
\hline butylated hydroxytoluene & $-1.7 \mathrm{E}-2$ \\
\hline$n$-butyl acrylate & $-1.5 \mathrm{E}-2$ \\
\hline epoxy, solid TGBAPPB & $-1.3 \mathrm{E}-2$ \\
\hline caustic soda beads & $-6.1 \mathrm{E}-3$ \\
\hline nitrile barrier resin & $-5.2 \mathrm{E}-3$ \\
\hline polymethyl methacrylate & $-3.5 \mathrm{E}-3$ \\
\hline polyacrylate latex & $-3.4 \mathrm{E}-3$ \\
\hline VDC-EA-MA copolymer & $-1.6 \mathrm{E}-3$ \\
\hline vinyl acetate-ethylene copolymer & $6.6 \mathrm{E}-4$ \\
\hline polyvinyl acetate & $7.9 \mathrm{E}-4$ \\
\hline acrylic acid, glacial & 0.25 \\
\hline$t$-butanol & 1.3 \\
\hline hydrogen peroxide & 2.8 \\
\hline refinery gas & 2.9 \\
\hline butadiene raffinate & 8.5 \\
\hline methyl ethyl ketone & 10. \\
\hline polyethylene terephthalate & 11 \\
\hline sec-butanol & 11 \\
\hline terephthalic acid & 19 \\
\hline$p$-xylene & 65 \\
\hline
\end{tabular}

Abbreviations:

ABS, acrylonitrile-butadiene-styrene; BPA, bisphenol A; DGEBPA, bisphenol A diglycidyl ether; SAN, styrene-acrylonitrile; TGBAPPB, tetraglycidyl amine from a,a'-bis(4-aminophenyl)p-diisopropylbenzene; VDC-EA-MA, vinylidene chloride-ethyl acetate-methacrylate. 